\title{
On a joint distribution of two successive surf parameters
}

\author{
D. Myrhaug ${ }^{1} \&$ H. Rue ${ }^{2}$ \\ ${ }^{1}$ Department of Marine Technology, \\ Norwegian University of Science and Technology, Trondheim, Norway \\ ${ }^{2}$ Department of Mathematical Sciences, \\ Norwegian University of Science and Technology, Trondheim, Norway
}

\begin{abstract}
A joint distribution of two successive surf parameters is provided, and it is represented by a bivariate lognormal distribution. Consequently the joint distribution of two successive breaker indices is represented by a bivariate lognormal distribution. The application of the surf parameter distribution is exemplified by estimating the probability of two successive breakers on slopes by using wave parameters corresponding to typical field conditions.
\end{abstract}

Keywords: bivariate lognormal distribution, surf zone, surf parameter, breaker index, breaking waves.

\section{Introduction}

The surf parameter, also often referred to as the surf similarity parameter or the Iribarren number, is used to characterize surf zone processes. It is given by the ratio between the slope of a beach or a structure and the square root of the wave steepness in deep water as introduced by Iribarren and Nogales [1] and used later by Battjes [2]. Shallow water regions where waves break are referred to as the surf zone, and the different breakers on slopes are defined and classified in terms of the surf parameter. It also appears that the surf parameter enters in many empirical and theoretical models for wave-induced phenomena in the surf zone. The breaking of waves is associated with large loss of energy. Within the surf zone along beaches the wave energy flux from offshore is dissipated into turbulence and heat, and consequently the wave height decreases towards the 
shoreline. Wave-breaking also results in strong currents along the shoreline and thereby affects the nearshore circulation. The high intensity of turbulence caused by wave-breaking is also responsible for the intense sediment transport in the surf zone. Wave run-up on beaches and coastal structures such as, e.g., breakwaters, seawalls and artificial reefs are characterized by using the surf parameter. Examples of the relevance and importance of the surf parameter are found in e.g. Herbich [3] and Silvester and Hsu [4].

Tayfun [5] presented a study on the development of approximate theoretical forms of the distributions of wave steepness and surf parameter. The approach is based on assuming the random wave process to be long crested and narrow banded. The results are compared with data from measurements at sea representing two severe storms. Both the wave steepness and the surf parameter are lognormal distributed. The resulting statistics for the surf parameter are applied to breakers at normal incidence on sloping beaches. As stated by Tayfun [5], the joint statistics of wave steepness with wave heights or crest heights, or the wave steepness with wave heights or crest heights above a specified threshold may be appropriate in formulating risks of e.g. capsizing of vessels, overtopping, and slamming forces on seawalls, etc. However, the wave steepness is of interest by itself, particularly in relation with many of the surf zone processes.

In a subsequent discussion Myrhaug and Fouques [6] pointed out that other data sets may result in other distributions. This is exemplified by using the data referred to by Tayfun [5]; the data used by Myrhaug and Kjeldsen [7, 8], Myrhaug and Rue [9], and Myrhaug and Kvålsvold [10]. These papers discuss various aspects of wave steepness statistics using data from a large population of waves obtained by waverider buoys at three different deep water locations on the Norwegian continental shelf. Myrhaug and Fouques [6] found that the wave steepness is Weibull distributed in the right tail and otherwise lognormal distributed, and that the surf parameter is lognormal distributed in the right tail and otherwise Fréchet distributed.

Myrhaug and Rue [9] used the Weibull model to study the statistics of two successive wave steepness parameters with the focus on steep waves, while Myrhaug and Kjeldsen [7], and Myrhaug [11] discussed the joint distribution of wave height and wave steepness. To our knowledge, no studies on the joint distribution of two successive surf parameters, i.e. the values of the surf parameter for two successive waves, are available in the open literature. This is the subject of the present paper, which should represent a useful tool for the assessment of various wave-induced phenomena in the surf zone.

Here the marginal distribution of the surf parameter is taken as the lognormal distribution, as found by Tayfun [5]. The joint distribution of two successive surf parameters is then represented by a two-dimensional lognormal distribution. The application of the results is illustrated by an example; the probability of two successive breaking waves on slopes are given. 


\section{Background}

The surf parameter is defined as $\xi=m / \sqrt{s}$ where $m=\tan \theta$ is the slope with an angle $\theta$ with the horizontal, $s=H /(g / 2 \pi) T^{2}$ is the wave steepness in deep water, $H$ is the wave height, $T$ is the wave period, and $g$ is the acceleration of gravity. In the forthcoming the surf parameter is normalized, i.e. $y=\xi / \xi_{r m s}$, by defining $\xi_{r m s}=m / \sqrt{s_{r m s}}$ where $s_{r m s}$ is the root-mean-square $(r m s)$ value of $s$, which will be discussed further in Section 4 . The basis for the present approach is that the marginal distribution of the normalized surf parameter follows the lognormal distribution with the probability density function $(p d f)$

$$
p(y)=\frac{1}{\sqrt{2 \pi} y \sigma_{z}} \exp \left[-\frac{\left(\ln y-\mu_{z}\right)^{2}}{2 \sigma_{z}^{2}}\right]
$$

where $\mu_{z}$ and $\sigma_{z}^{2}$ is the expected value and the variance, respectively, of $z=\ln y$. This is in accordance with the results in Tayfun [5]; further details are given in Section 4.

\section{Statistics of the joint behaviour of two successive surf parameters}

Now the joint distribution of the surf parameter for two successive waves is considered. There are several numbers of possible forms of two-dimensional distributions where marginal distributions are given by the lognormal distribution in Eq. (1). Let $y_{1}=\xi_{1} / \xi_{r m s}$ and $y_{2}=\xi_{2} / \xi_{r m s}$ denote the variables which are normalized with the same parameter $\xi_{r m s}$. Here $y_{1}$ and $y_{2}$ are associated with the first wave and the next succeeding wave, respectively. By introducing $z_{1}=\ln y_{1}$ and $z_{2}=\ln y_{2}$ in the two-dimensional Gaussian distribution in Eq. (A1) (see the Appendix), it can be transformed to a twodimensional distribution with the marginal distributions given by Eq. (1). This change of variables gives the following joint $p d f$ of normalized variables (see e.g. Johnson and Kotz [12])

$$
\begin{aligned}
& p\left(y_{1}, y_{2}\right)=\frac{1}{2 \pi y_{1} y_{2} \sigma_{z}^{2} \sqrt{1-\rho_{z_{1} z_{2}}^{2}}} . \\
& \exp \left[-\frac{\left(\ln y_{1}-\mu_{z}\right)^{2}+\left(\ln y_{2}-\mu_{z}\right)^{2}-2 \rho_{z_{1} z_{2}}\left(\ln y_{1}-\mu_{z}\right)\left(\ln y_{2}-\mu_{z}\right)}{2 \sigma_{z}^{2}\left(1-\rho_{z_{1} z_{2}}^{2}\right)}\right]
\end{aligned}
$$

where

$$
\begin{aligned}
& \mu_{z}=E\left[\ln y_{1}\right]=E\left[\ln y_{2}\right] \\
& \sigma_{z}^{2}=\operatorname{Var}\left[\ln y_{1}\right]=\operatorname{Var}\left[\ln y_{2}\right]
\end{aligned}
$$


The correlation coefficients $\rho_{z_{1} z_{2}}$ and $\rho_{y_{1} y_{2}}$ are related by

$$
\rho_{y_{1} y_{2}}=\frac{E\left[y_{1} y_{2}\right]-\mu_{y}^{2}}{\sigma_{y}^{2}}=\frac{e^{\rho_{z_{12} 2} \sigma_{z}^{2}}-1}{e^{\sigma_{z}^{2}}-1}
$$

by utilizing that

$$
\begin{aligned}
E\left[y_{1} y_{2}\right] & =\int_{0}^{\infty} \int_{0}^{\infty} y_{1} y_{2} p\left(y_{1}, y_{2}\right) d y_{1} d y_{2} \\
& =\exp \left[2 \mu_{z}+\sigma_{z}^{2}\left(1+\rho_{z_{1} z_{2}}\right)\right] \\
\mu_{y} & =e^{\mu_{z}+\frac{1}{2} \sigma_{z}^{2}} \\
\sigma_{y}^{2}=e^{2 \mu_{z}+\sigma_{z}^{2}} & \left(e^{\sigma_{z}^{2}}-1\right)
\end{aligned}
$$

Or, an alternative to Eq. (5) is

$$
\rho_{z_{1} z_{2}}=\frac{1}{\sigma^{2}} \ln \left[1+\rho_{y_{1} y_{2}}\left(e^{\sigma_{z}^{2}}-1\right)\right]
$$

The conditional $p d f$ of $y_{2}$ given $y_{1}$ is also lognormal distributed, given by (Johnson and Kotz [12])

$$
p\left(y_{2} \mid y_{1}\right)=\frac{p\left(y_{1}, y_{2}\right)}{p\left(y_{1}\right)}=\frac{1}{\sqrt{2 \pi} y_{2} \hat{\sigma}_{z}} \exp \left[-\frac{\left(\ln y_{2}-\hat{\mu}_{z}\right)^{2}}{2 \hat{\sigma}_{z}^{2}}\right]
$$

where

$$
\begin{gathered}
\hat{\mu}_{z}=\mu_{z}+\rho_{z_{1} z_{2}}\left(\ln y_{1}-\mu_{z}\right) \\
\hat{\sigma}_{z}{ }^{2}=\sigma_{z}^{2}\left(1-\rho_{z_{1} z_{2}}^{2}\right)
\end{gathered}
$$

The mean (expected) value of $y_{2}$ given $y_{1}$ is given by (Johnson and Kotz [12])

$$
\begin{aligned}
E\left[y_{2} \mid y_{1}\right] & =\int_{0}^{\infty} y_{2} p\left(y_{2} \mid y_{1}\right) d y_{2} \\
& =y_{1}^{\rho_{1122}} \exp \left[\frac{1}{2} \sigma_{z}^{2}\left(1-\rho_{z_{1} z_{2}}^{2}\right)+\mu_{z}\left(1-\rho_{z_{1} z_{2}}\right)\right]
\end{aligned}
$$

A quantity of interest is the probability of the surf parameter of a wave to be in an interval $y_{l}$ to $y_{h}$ when the surf parameter of the previous wave has been in the same interval. This probability is given as

$$
P=\frac{\int_{y_{l}}^{y_{h}} \int_{y_{l}}^{y_{h}} p\left(y_{1}, y_{2}\right) d y_{1} d y_{2}}{\int_{y_{l}}^{y_{h}} p\left(y_{1}\right) d y_{1}}=\frac{\int_{y_{l}}^{y_{h}}\left[\int_{y_{l}}^{y_{h}} p\left(y_{2} \mid y_{1}\right) d y_{2}\right] p\left(y_{1}\right) d y_{1}}{\Phi\left(u_{h}\right)-\Phi\left(u_{l}\right)}
$$

by using the relationship $p\left(y_{1}, y_{2}\right)=p\left(y_{2} \mid y_{1}\right) p\left(y_{1}\right)$, and where $\Phi$ denotes the standard Gaussian cumulative distribution function $(c d f)$, i.e. 


$$
\Phi(v)=\frac{1}{\sqrt{2 \pi}} \int_{-\infty}^{v} e^{-t^{2} / 2} d t
$$

and

$$
u_{l}=\frac{\ln y_{l}-\mu_{z}}{\sigma_{z}}, u_{h}=\frac{\ln y_{h}-\mu_{z}}{\sigma_{z}}
$$

The evaluation of the inner integral in the nominator of Eq. (14) follows by using Eqs. (10) to (12), giving

$$
P=\frac{\frac{1}{\sqrt{2 \pi}} \int_{u_{l}}^{u_{h}}\left[\Phi\left(\hat{u}_{h}\right)-\Phi\left(\hat{u}_{l}\right)\right] \exp \left(-\frac{1}{2} u_{1}^{2}\right) d u_{1}}{\Phi\left(u_{h}\right)-\Phi\left(u_{l}\right)}
$$

where

$$
\begin{gathered}
\hat{u}_{l}=\frac{u_{l}-\rho_{z_{1} z_{2}} u_{1}}{\sqrt{1-\rho_{z_{1} z_{2}}^{2}}}, \hat{u}_{h}=\frac{u_{h}-\rho_{z_{1} z_{2}} u_{1}}{\sqrt{1-\rho_{z_{1} z_{2}}^{2}}} \\
u_{1}=\frac{\ln y_{1}-\mu_{z}}{\sigma_{z}}
\end{gathered}
$$

\section{Example of application}

In this example the lognormal distribution of the surf parameter proposed by Tayfun [5] is adopted, given by the $p d f$

$$
p(\xi)=\frac{1}{\sqrt{2 \pi} \xi \sigma_{\ln \xi}} \exp \left[-\frac{\left(\ln \xi-\mu_{\ln \xi}\right)^{2}}{2 \sigma_{\ln \xi}^{2}}\right]
$$

where

$$
\mu_{\ln \xi}=\ln \left(\frac{2 m}{\sqrt{\alpha}}\right), \quad \sigma_{\ln \xi}^{2}=\frac{1}{4} \ln \left(\frac{4}{\pi}\right)
$$

and $\alpha$ is a parameter related to the wave steepness of the sea state $s_{r m s}$. From Tayfun [5] it appears that his theoretical value of the sea state steepness parameter, $s_{r m s}=0.318 \alpha$, is very close to $s_{r m s}=17.6 H_{s} / 4 g T_{z}^{2}$ used by Myrhaug and Rue [9]; thus giving $\alpha=1.41 H_{s} / T_{z}^{2}$. Here $H_{s}$ is the significant wave height and $T_{z}$ is the mean zero-crossing wave period.

Now a change of variables from $\xi$ to $y=\xi / \xi_{r m s}$ gives the lognormal $p d f$ in Eq. (1) with $\mu_{z}=\mu_{\ln \xi}-\ln \xi_{r m s}=\ln (2 \sqrt{0.318})$ and $\sigma_{z}^{2}=\sigma_{\ln \xi}^{2}$, giving

$$
\mu_{z}=0.120, \sigma_{z}=0.246
$$

Figure 1(a) shows the mean (expected) value of $y_{2}=\xi_{2} / \xi_{\text {rms }}$ given $y_{1}=\xi_{1} / \xi_{r m s}$ versus $y_{1}$ according to Eq. (13) for a range of $\rho_{z_{1} z_{2}}$ values from 0 to 0.9 . From Fig. 1(a) it appears that $E\left[y_{2} \mid y_{1}\right]$ approaches $y_{1}$ as $\rho_{z_{1} z_{2}}$ 
increases. It should be noted that $E\left[y_{2} \mid y_{1}\right]=y_{1}$ for $\rho_{z_{1} z_{2}}=1$; see Eq. (13). For zero correlation the mean value of $y_{2}$ given $y_{1}$ is always constant, i.e. Eq. (13) reduces to Eq. (7).
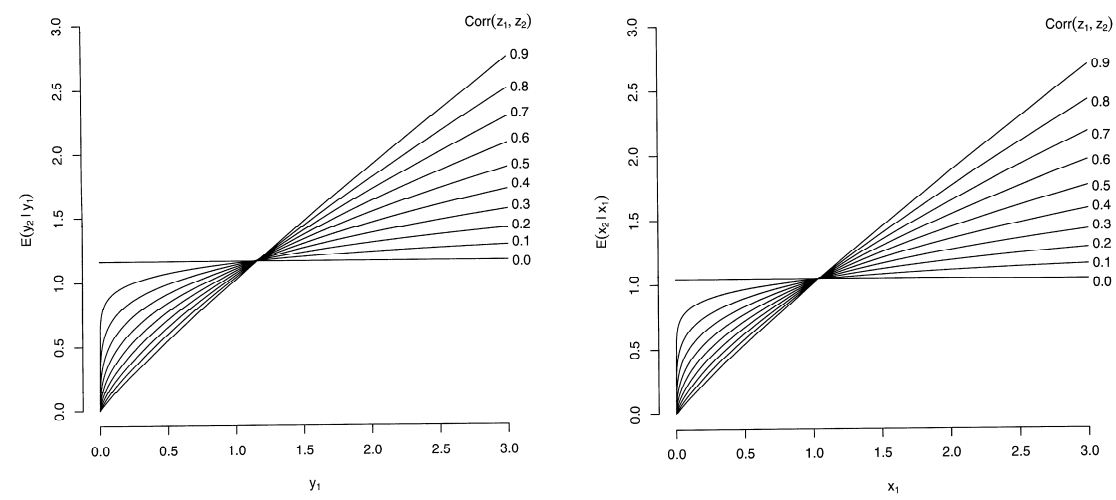

Figure 1: (a) (left) Conditional expected value of $y_{2}=\xi_{2} / \xi_{\text {rms }}$ given $y_{1}=\xi_{1} / \xi_{\text {rms }}$ versus $y_{1}$ for different values of $\rho_{z_{1} z_{2}}=\operatorname{Corr}\left(z_{1}, z_{2}\right)$. (b) (right) Conditional expected value of $x_{2}=h_{2 b} / h_{b r m s}$ given $x_{1}=h_{1 b} / h_{b r m s}$ versus $x_{1}$ for different values of $\rho_{z_{1} z_{2}}=\operatorname{Corr}\left(z_{1}, z_{2}\right)$.

Furthermore, a sea state specified by $H_{s}=7$ metres and $T_{z}=7$ seconds is chosen, representing a "steep" sea state which has been measured at a deep water location on the Norwegian continental shelf (Krogstad [13]). Thus, $s_{r m s}=0.064$ and $\alpha=0.20$, giving $\xi_{\text {rms }}=3.95 \mathrm{~m}$.

Moreover, in this example breaking waves on slopes will be considered. Types of breaking waves are defined in terms of the surf parameter, classified as (see e.g. Tayfun [5])

$\begin{array}{ll}\text { spilling if } & \xi \leq 0.5 \\ \text { plunging for } & 0.5<\xi \leq 3 \\ \text { collapsing for } & 3<\xi \leq 3.5 \\ \text { surging if } & 3.5<\xi\end{array}$

Thus, by taking $y_{1}=\xi_{1} / \xi_{r m s}, y_{2}=\xi_{2} / \xi_{r m s}$ and $\mu_{z}, \sigma_{z}$ from Eq. (22), Eqs. (16) to (19) can be used to calculate the probability of two successive breaking waves on slopes.

Figure 2 shows the probability $P$ of two successive spilling breakers versus the correlation coefficient $\rho_{z_{1} z_{2}}$ for the slopes $\mathrm{m}=1 / 10,1 / 5,1 / 4,1 / 3,1 / 2$. 


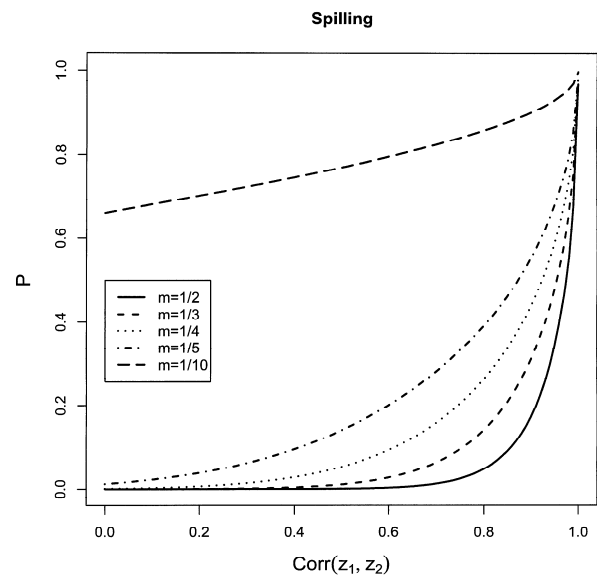

Figure 2: The probability $(P)$ of two successive spilling breakers versus $\rho_{z_{1} z_{2}}=\operatorname{Corr}\left(z_{1}, z_{2}\right)$ for different slopes $\mathrm{m}$.

Similar results are shown in Figures 3, 4 and 5 for plunging, collapsing and surging breakers, respectively.

From Figs. 2 to 5 it appears that $P$ increases as $\rho_{z_{1} z_{2}}$ increases for a given slope, which is physically sound. Moreover, from Fig. 2 it appears that $P$ decreases as the slope increases for a given value of $\rho_{z_{1} z_{2}}$, which a priori is not quite obvious. However, it can be demonstrated by considering the results for $\rho_{z_{1} z_{2}}=0$, i.e. when $y_{1}$ and $y_{2}$ are statistically independent. Then the marginal $p d f$ of $y_{1}$ (and $y_{2}$ ) is given by Eq. (1) and $\mu_{z}, \sigma_{z}$ from Eq. (22), and the $p d f$ of $\xi=\xi_{1}\left(\right.$ or $\left.\xi_{2}\right)$ for $\xi_{r m s}=3.95 \mathrm{~m}$ is shown in Fig. 6 for the same slopes $\mathrm{m}$ as in Figs. 2 to 5. From Fig. 6 it appears that the probability of a spilling breaker for a given slope (i.e. given by the area under the $p d f$ for the slope considered corresponding to $0<\xi \leq 0.5$ ), is largest for $\mathrm{m}=1 / 10$ and decreases as the slope increases. This will also be the case for other values of $\rho_{z_{1} z_{2}}$, and consequently the results are as shown in Fig. 2.

From Fig. 3 it appears that the probability of two successive plunging breakers for a given value of $\rho_{z_{1} z_{2}}$ increases for the slope in the order $\mathrm{m}=1 / 10$, $1 / 2,1 / 5,1 / 3,1 / 4$; for the three latter values the differences are very small. The understanding of this is supported by the results for $\rho_{z_{1} z_{2}}=0$ in Fig. 6; for $0.5<\xi \leq 3.0$ it is observed that the area under the $p d f$ is smallest for $\mathrm{m}=1 / 10$ and that it increases for the slope in the order referred to in the discussion of Fig. 3. 


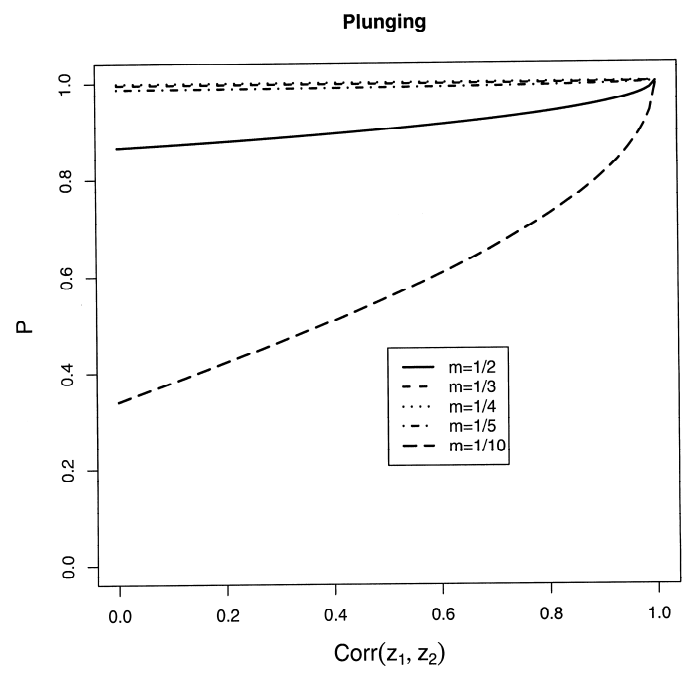

Figure 3: The probability $(P)$ of two successive plunging breakers versus $\rho_{z_{1} z_{2}}=\operatorname{Corr}\left(z_{1}, z_{2}\right)$ for different slopes $\mathrm{m}$.

The results for the collapsing and surging breakers in Figs. 4 and 5, respectively, are similar; it appears that the probability of two successive collapsing and surging breakers increases as the bed slope increases for a given value of $\rho_{z_{1} z_{2}}$, which is supported by the results in Fig. 6 for $\rho_{z_{1} z_{2}}=0$.

Collapsing

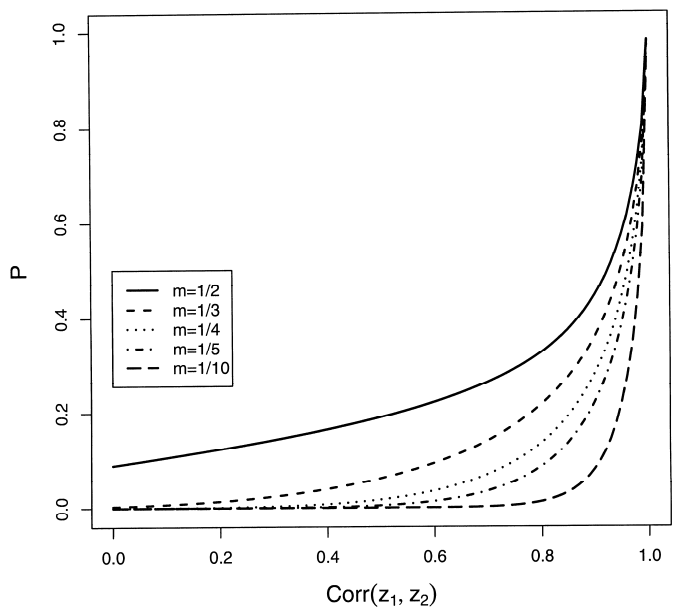

Figure 4: The probability $(P)$ of two successive collapsing breakers versus $\rho_{z_{1} z_{2}}=\operatorname{Corr}\left(z_{1}, z_{2}\right)$ for different slopes $\mathrm{m}$. 


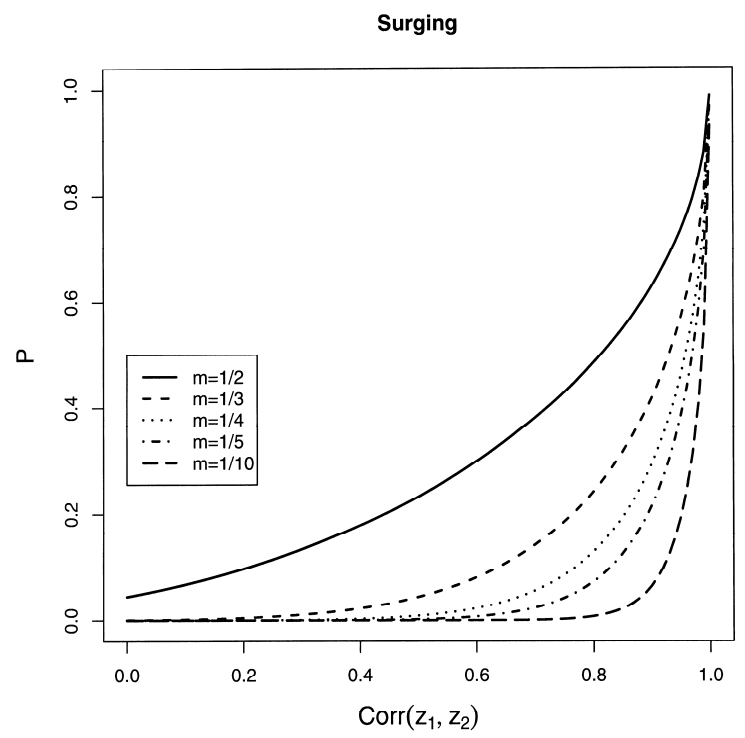

Figure 5: The probability $(P)$ of two successive surging breakers versus $\rho_{z_{1} z_{2}}=\operatorname{Corr}\left(z_{1}, z_{2}\right)$ for different slopes $\mathrm{m}$.

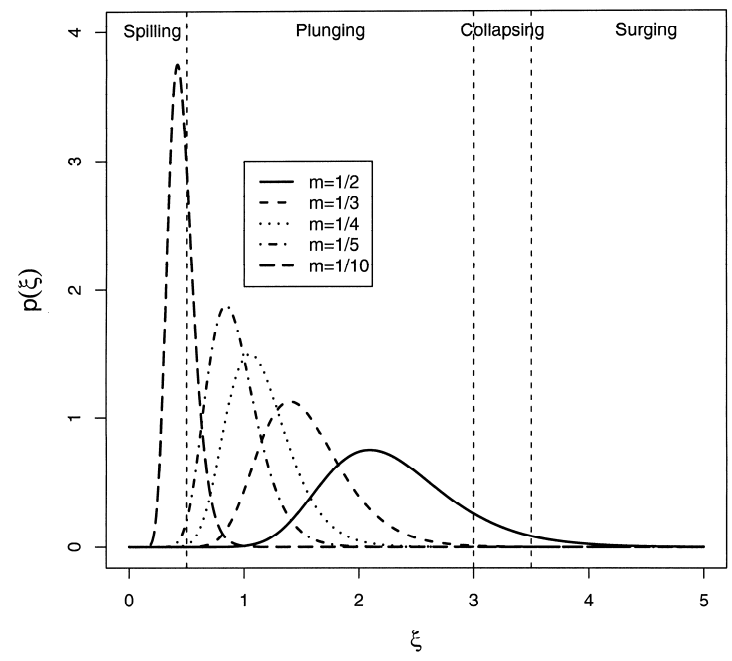

Figure 6: The $p d f$ of the surf parameter $\xi=\xi_{1}\left(\right.$ or $\left.\xi_{2}\right)$ for $\xi_{r m s}=3.95 \mathrm{~m}$ and different slopes $\mathrm{m}$. 
Overall, the results given in this example appears to be physically sound, although they are valid for the particular sea state chosen. However, validation with data is required before a conclusion can be drawn on the ability of the present approach to describe measured wave data. Thus the results should be taken as tentative, but in the meantime the present distribution of two successive surf parameters should serve the purpose of being a useful tool for making assessments of wave phenomena in the surf zone, i.e. to obtain an estimate of two extreme successive wave events in the surf zone.

\section{Statistics of the joint behaviour of two successive breaker indices}

The breaker index $h_{b}$ is another frequently used quantity in coastal work, which is closely related to the surf parameter. It is defined as the ratio between the wave height $H_{b}$ and the water depth $d_{b}$ at breaking. Many empirical relationships exist for $h_{b}$; one is related to the surf parameter in the form $h_{b} \equiv H_{b} / d_{b}=a \xi^{c}$ (Tayfun [5]), where $a$ and $c$ are empirical coefficients. In the forthcoming the breaker index is normalized, i.e. $x=h_{b} / h_{b r m s}$, by defining $h_{b r m s}=a \xi_{r m s}^{c}$ where $\xi_{r m s}$ is defined in Section 2, giving $x=\left(\xi / \xi_{r m s}\right)^{c}=y^{c}$. The $p d f$ of $x$ is obtained from Eq. (1) by a change of variable from $y$ to $x$, taking the form

$$
p(x)=\frac{1}{\sqrt{2 \pi} x \sigma} \exp \left[-\frac{(\ln x-\mu)^{2}}{2 \sigma^{2}}\right]
$$

where

$$
\mu=c \mu_{z}, \sigma^{2}=\left(c \sigma_{z}\right)^{2}
$$

are the mean value and the variance, respectively, of $\ln x$.

Thus, the statistics of the joint behaviour of two successive breaker indices $x_{1}=h_{b 1} / h_{b r m s}$ and $x_{2}=h_{b 2} / h_{b r m s}$ normalized with the same parameter $h_{b r m s}$ follow by utulizing the results in Section 3. More specifically it follows that: $p\left(x_{1}, x_{2}\right)$ is given in Eq. (2); $p\left(x_{2} \mid x_{1}\right)$ in Eqs. (10) to (12); $E\left[x_{2} \mid x_{1}\right]$ in Eq. (13), by replacing $y_{1}, y_{2}, \mu_{z}, \sigma_{z}$ with $x_{1}, x_{2}, c \mu_{z}, c \sigma_{z}$. Moreover, $\rho_{x_{1} z_{2}}$ is given in Eq. (5) (or alternatively $\rho_{z_{1} z_{2}}$ in Eq. (9)) by replacing $y_{1}, y_{2}, \sigma_{z}$ with $x_{1}, x_{2}, c \sigma_{z}$; this is obtained by utilizing the results in Eqs. (6) to (8) by in addition replacing $y, \mu_{z}$ with $x, c \mu_{z}$. The results in Eqs. (14) to (19) can be re-arranged accordingly to be valid for two successive breaker indices.

Figure 1(b) shows the mean value of $x_{2}=h_{b 2} / h_{b r m s}$ given $x_{1}=h_{b 1} / h_{b r m s}$ versus $x_{1}$ for a range of $\rho_{z_{1} z_{1}}$ values from 0 to 0.9 according to Eq. (13) by replacing $y_{1}, y_{2}, \mu_{z}, \sigma_{z}$ with $x_{1}, x_{2}, c \mu_{z}, c \sigma_{z}$, and $(a, c)=(1.20,0.27)$ from Kaminsky and Kraus [14]. The results are similar to those given in Fig. 1(a) for the surf parameter except for the shift of the values. Results for the breaker index 
will not be elaborated further here since they will be similar to those presented for the surf parameter.

\section{Summary}

A joint distribution of two successive surf parameters is provided, and it is represented by a bivariate lognormal distribution. Consequently the joint distribution of two successive breaker indices is represented by a bivariate lognormal distribution. The application of the surf parameter distribution is exemplified to estimate the probability of two successive breakers on slopes; spilling, plunging, collapsing and surging breakers, by using wave parameters corresponding to typical field conditions. Overall, these results appear to be physically sound, although they are valid for the particular sea state chosen. The results should be taken as tentative, because validation with data is required before a conclusion can be drawn on the ability of the present approach to describe measured wave data. However, in the meantime the bivariate lognormal distribution of two successive surf parameters should serve the purpose as a useful tool for making assessments of wave phenomena in the surf zone, i.e. to obtain an estimate of two extreme successive wave events in the surf zone.

\section{Appendix}

The joint $p d f$ of two Gaussian random variables $z_{1}$ and $z_{2}$ with the same mean value $\mu_{z}$ and variance $\sigma_{z}^{2}$, is given by (Bury [15])

$$
\begin{aligned}
& p\left(z_{1}, z_{2}\right)=\frac{1}{2 \pi \sigma_{z}^{2} \sqrt{1-\rho_{z_{1} z_{2}}^{2}}} . \\
& \exp \left[-\frac{\left(z_{1}-\mu_{z}\right)^{2}+\left(z_{2}-\mu_{z}\right)^{2}-2 \rho_{z_{1} z_{2}}\left(z_{1}-\mu_{z}\right)\left(z_{2}-\mu_{z}\right)}{2 \sigma_{z}^{2}\left(1-\rho_{z_{1} z_{2}}^{2}\right)}\right]
\end{aligned}
$$

where the correlation coefficient $\rho_{z_{1} z_{2}}$ is given as

$$
\rho_{z_{1} z_{2}} \equiv \frac{\operatorname{Cov}\left[z_{1}, z_{2}\right]}{\sigma_{z}^{2}}=\frac{E\left[z_{1} z_{2}\right]-\mu_{z}^{2}}{\sigma_{z}^{2}}
$$

\section{References}

[1] Iribarren, C.R. \& Nogales, C., Protection des ports, Sect. 2. Comm. 4, $17^{\text {th }}$ Int. Nav. Cong. Lisbon, pp. 31-80, 1949.

[2] Battjes, J.A., Surf similarity. Proceedings $14^{\text {th }}$ Int. Conf. on Coastal Engineering, ASCE, New York, Vol. 1, pp. 466-479, 1974.

[3] Herbich, J.B., Handbook of Coastal and Ocean Engineering. Volume 1. Wave Phenomena and Coastal Structures. Gulf Publishing Co., Houston, Texas, 1990. 
[4] Silvester, R. \& Hsu, J.R.C., Coastal Stabilization, World Scientific, Singapore, 1997.

[5] Tayfun, M.A., Distributions of wave steepness and surf parameter. $J$. Waterway, Port, Coastal, Ocean Eng., 132(1), pp. 1-9, 2006.

[6] Myrhaug, D. \& Fouques, S., Discussion of "Distributions of wave steepness and surf parameter" by M. Aziz Tayfun. J. Waterway, Port, Coastal, Ocean Eng., 133 (3), pp. 242-243, 2007.

[7] Myrhaug, D. \& Kjeldsen, S.P., Parametric modelling of joint probability density distributions for steepness and asymmetry in deep water waves. Appl. Ocean Res. 6(4), pp. 207-220, 1984.

[8] Myrhaug, D. \& Kjeldsen, S.P., Predictions of occurrences of steep and high waves in deep water. J. Waterway, Port, Coastal, Ocean Eng., 113(2), pp. 122-138, 1987.

[9] Myrhaug, D. \& Rue, H., Joint distribution of successive wave steepness parameters. J. Offshore Mech. Arct. Eng. 115(3), pp. 191-195, 1993.

[10] Myrhaug, D. \& Kvålsvold, J., Comparative study of joint distributions of primary wave characteristics. J. Offshore Mech. Arct. Eng., 117(2), pp. 91-98, 1995.

[11] Myrhaug, D., Statistics of steep waves in deep water. J. Marine Environ. Eng. 1(2), pp. 161-173, 1994.

[12] Johnson, N.L. \& Kotz, S., Distributions in Statistics: Continuous Multivariate Distributions, John Wiley \& Sons, New York, 1972.

[13] Krogstad, H.E., Height and period distributions of extreme waves. Appl. Ocean Res., 7(3), pp. 158-165, 1985.

[14] Kaminsky, G.M. \& Kraus, N.C., Evaluation of depth-limited wave breaking criteria. Proceedings $2^{\text {nd }}$ Int. Symp. on Wave Measurements and Analysis, ASCE, New York, pp. 180-193, 1994.

[15] Bury, K.V., Statistical Models in Applied Science. John Wiley \& Sons, New York, 1975. 